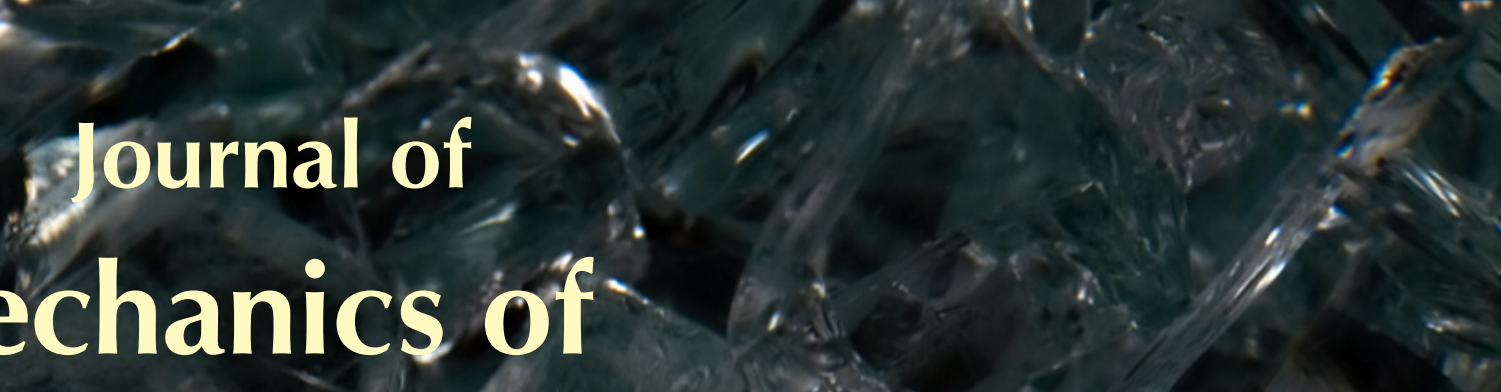

Materials and Structures

Mechanics of

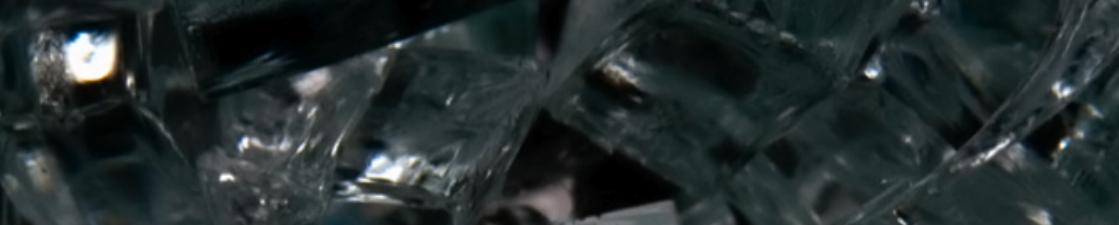

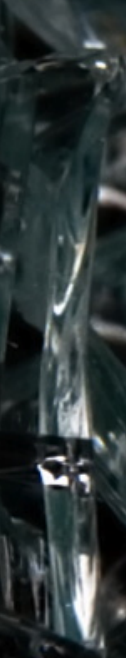

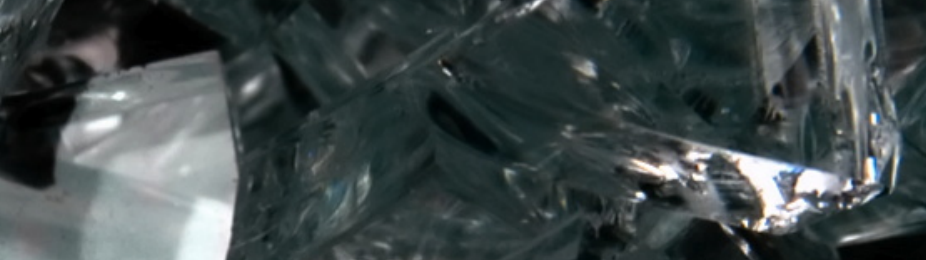

$y=1$

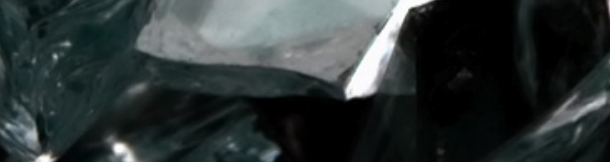

is

FREE VIBKATION OF A SIMULATION CANDU NUCLEAR S FUELCBUNDLE STRUCTURE INSIDE A TUBE

"Xuan Zhang and Shudông Yu
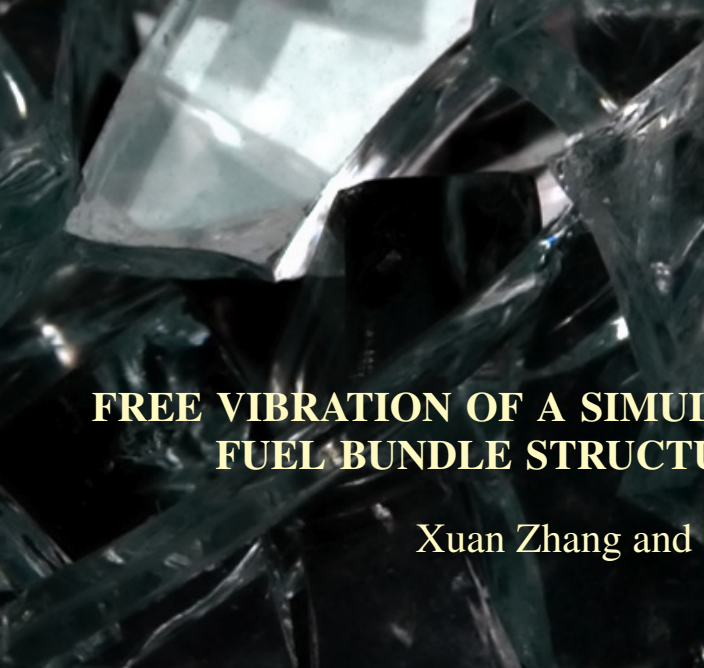

L.

2

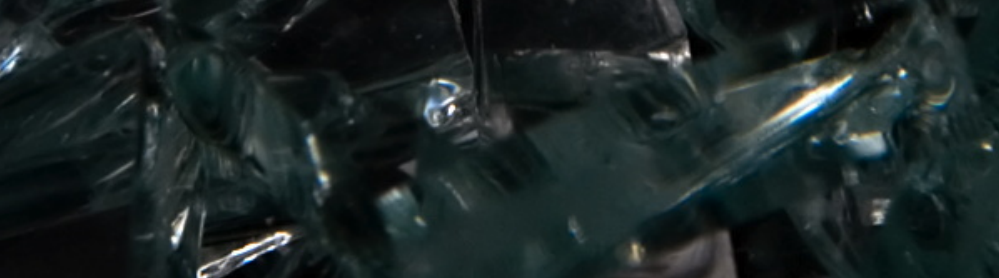

Volume 6, No. 7-8

Septembe October 2011

(1).$-n$ 


\title{
FREE VIBRATION OF A SIMULATION CANDU NUCLEAR FUEL BUNDLE STRUCTURE INSIDE A TUBE
}

\author{
Xuan Zhang And Shudong Yu
}

\begin{abstract}
This paper presents a numerical rigid-elasto model for vibration of a simulation nuclear fuel bundle structure confined in a circular tube. The model is developed using the finite element method combined with the floating frame formulation. The nonlinear dynamic equations are derived using the Lagrange equations. Small-amplitude vibration about the static equilibrium position is obtained through linearization. Numerical results show that the fundamental mode is a rocking-like mode, in which rigid body translation and rotation are coupled with elastic deformations. Gravity is found to reduce the frequency of the fundamental mode without affecting the higher modes. Experiments are conducted for a single fuel bundle structure to validate the numerical results.
\end{abstract}

\section{Introduction}

Horizontally placed CANDU ${ }^{1}$ fuel bundles in a circular tube are used in the Canadian nuclear industry. During operation, the fuel bundles experience small-amplitude flow-induced vibration, which can result in significant wear to the supporting structures over a period of time.

Static deformations of fuel bundle structures have been investigated in [Cho et al. 2000; Horhoianu and Ionescu 2006]. Dynamic models of a horizontally placed rod bundle sitting on the inner surface of a tube have not been seen before in the literature.

A 43-element simulation fuel bundle is shown in Figure 1 on the next page. The bundle has 43 rods distributed in 3 rings and at the center. The rods are interconnected by two endplates. Each endplate consists of 3 circular rings and 16 ribs/webs. The weight of the bundle is supported by the bearing pads on the bottom rods in the outer ring, as shown on the right. Ideally there are four bearing pads in contact with the tube surface and supporting the bundle weight. The radial gaps between the tube surface and the bearing pads on the rods next to the bottom rods are about $30-50 \mu \mathrm{m}$, which permits small-amplitude bundle vibration without impacting the supporting structure. This smallamplitude vibration results in a relatively large motion in the top parts of the bundle due to the large bundle diameter. When disturbed or excited, the bundle vibrates about its equilibrium position.

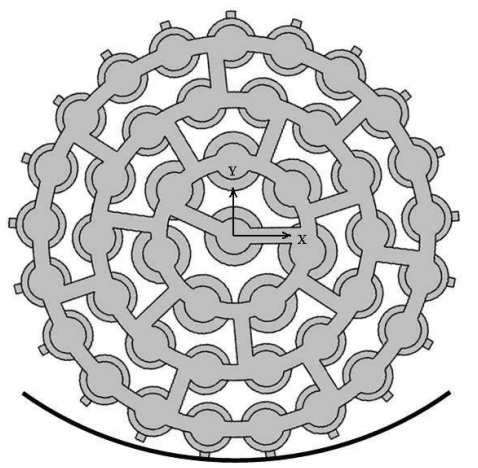

Fuel channel inspections at the Darlington nuclear station indicated that bundle vibrations, for example, rocking, induced by the coolant flow were responsible for the fretting between the pressure tube spacer sleeve and the inlet bundle bearing pads [Judah 1992]. Modeling the bundle vibration requires

Keywords: multibody dynamics, floating frame, fuel bundle structure, vibration.

${ }^{1}$ CANadian Deuterium and Uranium, a registered trademark of Atomic Energy of Canada Ltd. 
formulation of the rigid-elastic motions of a 3D bundle structure. The superimposition method [Schwab and Meijaard 2002] and the floating frame formulation [Shabana 2005] have been used to deal with rigid and elastic motions of a 3D body.

Extending from [Zhang and Yu 2010a], this paper presents a numerical procedure for modeling the rod bundles using the floating frame formulation. A rigid-elastodynamic model is established and applied to the study of the fundamental mode of a single bundle. The influence of gravity is discussed based on the simulation results. The simulation results are compared to experimental results.

\section{Finite element model in the floating frame formulation}

The floating frame formulation used in multibody dynamics is employed to couple the rigid body motion and the elastic deformation of the bundle. Nonlinear equations of motion are obtained for the bundle vibration, and then simplified to a set of linear equations for the small-amplitude vibrations about the equilibrium.

As shown in Figure 1, left, a global inertia frame is chosen in such a way that the $X$-axis is horizontal and the $Y$-axis is vertical. The origin of the global frame is chosen to be the center of the upstream endplate. To describe the rigid body motion, a reference frame $x-y-z$ is also defined in the figure. The frame is rigidly attached to the mass center of the bundle so that it moves and rotates with the bundle. This frame is regarded as the body frame of the bundle.

The bundle is discretized using two types of finite elements. The rods are modeled using a three-node higher-order beam element in conjunction with Euler-Bernoulli theory [Meirovitch 2001]. The endplates are modeled using a special nine-node isoparametric plate element [Yu and Wen 2007] in conjunction with the third-order thick plate theory of [Reddy 1984]. The finite element model is shown in Figure 1, right. The endplate is regarded as massless because its mass is significantly smaller than the mass of the rods. Therefore the stiffness of the endplate can be condensed using static substructuring. A superelement can then be achieved for each endplate using the procedures described in [Zhang and Yu 2010b].

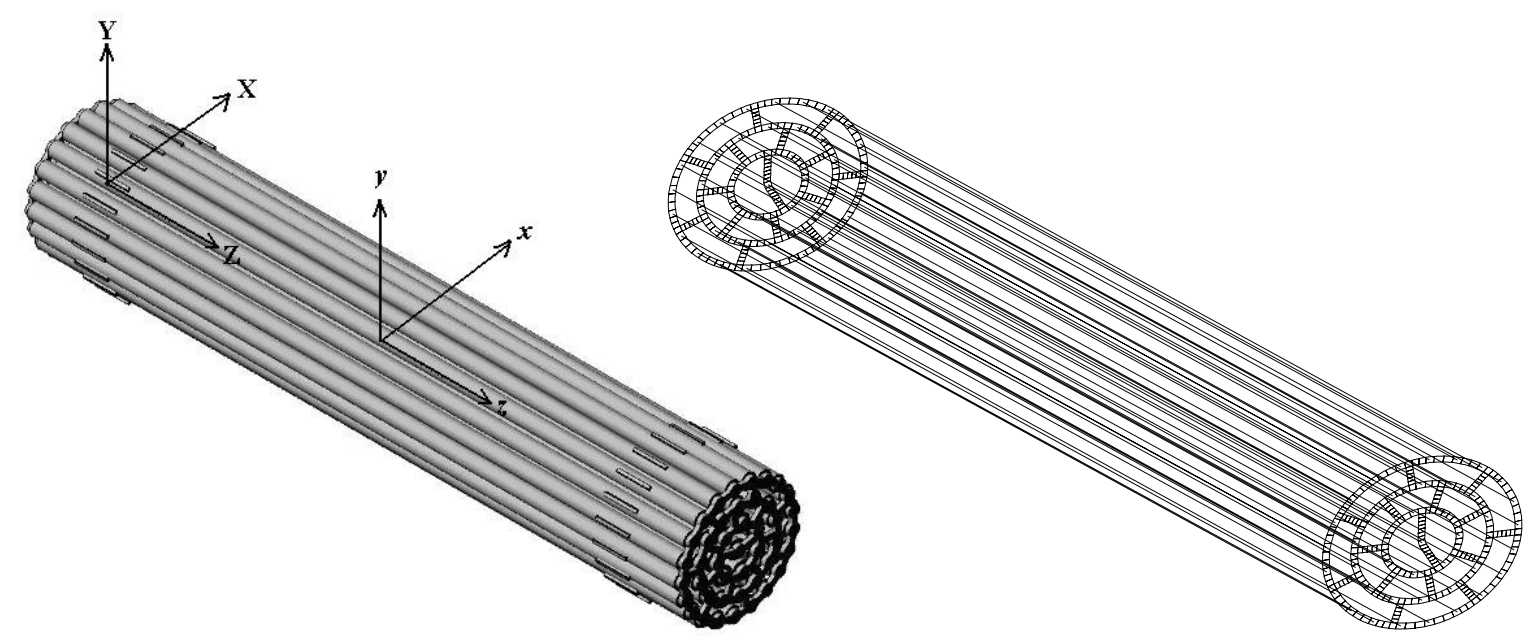

Figure 1. Rod bundle: isoparametric view (left) and finite-element mesh (right). 
Two sets of degrees of freedom (DOFs) are considered in this model. The first set is the rigid body (body frame) displacement including translations $\boldsymbol{u}_{c}$ and rotations $\boldsymbol{\phi}$ measured in the global frame $X-Y-Z$. The second set is the deformation-induced displacement $\boldsymbol{u}_{f}$ measured in the body frame. In the floating frame formulation [Shabana 2005], the displacement of an arbitrary material point on the bundle can be expressed as

$$
\boldsymbol{u}=\boldsymbol{u}_{c}+\left[\boldsymbol{A}(\boldsymbol{\phi})-\boldsymbol{A}_{0}\right] \boldsymbol{x}+\boldsymbol{A}(\boldsymbol{\phi}) \boldsymbol{u}_{f},
$$

where $A(\phi)=A_{3} A_{2} A_{1}$ is the Euler rotation matrix,

$$
\boldsymbol{A}_{3}=\left[\begin{array}{ccc}
\cos \varphi_{3} & -\sin \varphi_{3} & 0 \\
\sin \varphi_{3} & \cos \varphi_{3} & 0 \\
0 & 0 & 1
\end{array}\right], \quad \boldsymbol{A}_{2}=\left[\begin{array}{ccc}
\cos \varphi_{2} & 0 & \sin \varphi_{2} \\
0 & 1 & 0 \\
-\sin \varphi_{2} & 0 & \cos \varphi_{2}
\end{array}\right], \quad \boldsymbol{A}_{1}=\left[\begin{array}{ccc}
1 & 0 & 0 \\
0 & \cos \varphi_{1} & -\sin \varphi_{1} \\
0 & \sin \varphi_{1} & \cos \varphi_{1}
\end{array}\right] .
$$

$\varphi_{1}, \varphi_{2}$, and $\varphi_{3}$ are three Euler angles. $\boldsymbol{A}^{0}$ is the initial value of $\boldsymbol{A}$ and is equal to the identity matrix $\boldsymbol{I}$; $\boldsymbol{u}_{f}$ can be further expressed in terms of the finite element nodal displacement $\overline{\boldsymbol{u}}_{f}$ as

$$
\boldsymbol{u}_{f}=\boldsymbol{t}^{T} \boldsymbol{N}(x) \boldsymbol{T} \overline{\boldsymbol{u}}_{f},
$$

where $N(x)$ is the shape function of the beam element, while $\boldsymbol{t}$ and $\boldsymbol{T}$ are the transformation matrices between the element local frame and the body frame coordinates for the material point and the nodal DOFs, respectively. Vector $\boldsymbol{x}$ is the distance vector from the body frame origin to the material point measured in the body frame. Vector $\boldsymbol{x}$ can be expressed as $\boldsymbol{x}=\boldsymbol{x}_{E}^{(b)}+\boldsymbol{t}^{T} \boldsymbol{x}^{(e)}$, where $\boldsymbol{x}_{E}^{(b)}$ is the distance vector from the body frame origin to the element local frame origin measured in the body frame and $\boldsymbol{x}^{(e)}$ is the distance vector from the element local frame origin to the material point measured in the element local frame.

The velocity of the arbitrary material point is

$$
\dot{\boldsymbol{u}}=\dot{\boldsymbol{u}}_{c}+\dot{\boldsymbol{A}}\left(\boldsymbol{x}+\boldsymbol{u}_{f}\right)+\boldsymbol{A} \dot{\boldsymbol{u}}_{f}=\left[\begin{array}{ll}
\boldsymbol{L}_{r} & \tilde{\boldsymbol{L}}_{f}
\end{array}\right]\left\{\begin{array}{c}
\dot{\boldsymbol{u}}_{r} \\
\dot{\overline{\boldsymbol{u}}}_{f}
\end{array}\right\}=\boldsymbol{L} \dot{\boldsymbol{q}}
$$

where

$$
\begin{aligned}
& \boldsymbol{q}=\left\{\begin{array}{ll}
\boldsymbol{u}_{r}^{T} & \overline{\boldsymbol{u}}_{f}^{T}
\end{array}\right\}^{T}, \quad \boldsymbol{u}_{r}=\left\{\begin{array}{ll}
\boldsymbol{u}_{c}^{T} & \boldsymbol{\phi}^{T}
\end{array}\right\}^{T}, \quad \boldsymbol{L}_{r}=\left[\begin{array}{ll}
\boldsymbol{I} & \boldsymbol{B}
\end{array}\right], \quad \tilde{\boldsymbol{L}}_{f}=[\tilde{\boldsymbol{A}}]=\left[\begin{array}{ll}
\boldsymbol{A} \boldsymbol{t}^{T} \boldsymbol{N}(x) \boldsymbol{T} \overline{\boldsymbol{u}}_{f}
\end{array}\right],
\end{aligned}
$$

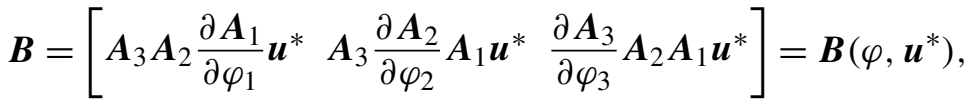

$$
\begin{aligned}
& \boldsymbol{u}^{*}=\boldsymbol{x}+\boldsymbol{u}_{f}=\boldsymbol{x}+\boldsymbol{t}^{T} \boldsymbol{N}(x) \boldsymbol{T} \overline{\boldsymbol{u}}_{f} .
\end{aligned}
$$

The kinetic energy of the system can then be expressed as

$$
T=\int_{V} \frac{1}{2} \rho \dot{\boldsymbol{u}}^{T} \dot{\boldsymbol{u}} d V=\frac{1}{2}\left\{\begin{array}{l}
\dot{\boldsymbol{u}}_{r} \\
\dot{\boldsymbol{u}}_{f}
\end{array}\right\}^{T}\left[\begin{array}{cc}
\boldsymbol{M}_{r r} & \boldsymbol{M}_{r f} \\
\boldsymbol{M}_{f r} & \boldsymbol{M}_{f f}
\end{array}\right]\left\{\begin{array}{c}
\dot{\boldsymbol{u}}_{r} \\
\dot{\overline{\boldsymbol{u}}}_{f}
\end{array}\right\}=\frac{1}{2} \dot{\boldsymbol{q}}^{T} \boldsymbol{M} \dot{\boldsymbol{q}}
$$

where

$$
\boldsymbol{M}_{r r}=\int_{V} \rho \boldsymbol{L}_{r}^{T} \boldsymbol{L}_{r} d V, \quad \boldsymbol{M}_{r f}=\int_{V} \rho \boldsymbol{L}_{r}^{T} \tilde{\boldsymbol{L}}_{f} d V, \quad \boldsymbol{M}_{f r}=\int_{V} \rho \tilde{\boldsymbol{L}}_{f}^{T} \boldsymbol{L}_{r} d V, \quad \boldsymbol{M}_{f f}=\int_{V} \rho \tilde{\boldsymbol{L}}_{f}^{T} \tilde{\boldsymbol{L}}_{f} d V .
$$


The potential energy of the system is simply the summation of the elastic potential energy $V_{e}$ and the gravity potential energy $V_{g}$ :

$$
V=V_{e}+V_{g}, \quad V_{e}=\frac{1}{2}\left\{\begin{array}{l}
\boldsymbol{u}_{r} \\
\overline{\boldsymbol{u}}_{f}
\end{array}\right\}^{T}\left[\begin{array}{cc}
\mathbf{0} & \mathbf{0} \\
\mathbf{0} & \boldsymbol{K}_{f f}
\end{array}\right]\left\{\begin{array}{l}
\boldsymbol{u}_{r} \\
\overline{\boldsymbol{u}}_{f}
\end{array}\right\}=\frac{1}{2} \boldsymbol{q}^{T} \boldsymbol{K} \boldsymbol{q}, \quad V_{g}=\int_{V} \rho g\left[\begin{array}{lll}
0 & 1 & 0
\end{array}\right] \boldsymbol{r} d V,
$$

where $\boldsymbol{K}_{f f}$ is the stiffness matrix obtained using the finite element method for the bundle and $\boldsymbol{r}$ is the distance vector from the global origin to the arbitrary material point.

The bundle is assumed to be constrained at the four bearing pad locations. The bundle cannot move due to the large weight and the frictional force between the bundle and the hosting tube. It is justified to consider the nodes as simply supported at the four bearing pad locations when small oscillatory motion is studied. Therefore, the following constraint conditions apply:

$$
\boldsymbol{u}^{(i)}=\boldsymbol{u}_{c}+\left[\boldsymbol{A}(\boldsymbol{\phi})-\boldsymbol{A}^{0}\right] \boldsymbol{x}^{(i)}+\boldsymbol{A}(\boldsymbol{\phi}) \overline{\boldsymbol{u}}_{f}^{(i)}=\mathbf{0},
$$

where superscript $(i)$ represents the $i$-th constrained node $(i=1, \ldots, 4)$.

According to the free and constrained DOFs, (1) and (2) can be partitioned

$$
T=\frac{1}{2}\left\{\begin{array}{c}
\dot{\boldsymbol{u}}_{r} \\
\dot{\overline{\boldsymbol{u}}}_{f c} \\
\dot{\overline{\boldsymbol{u}}}_{f i}
\end{array}\right\}^{T}\left[\begin{array}{lll}
\boldsymbol{M}_{11} & \boldsymbol{M}_{12} & \boldsymbol{M}_{13} \\
\boldsymbol{M}_{21} & \boldsymbol{M}_{22} & \boldsymbol{M}_{23} \\
\boldsymbol{M}_{31} & \boldsymbol{M}_{32} & \boldsymbol{M}_{33}
\end{array}\right]\left\{\begin{array}{c}
\dot{\boldsymbol{u}}_{r} \\
\dot{\overline{\boldsymbol{u}}}_{f c} \\
\dot{\overline{\boldsymbol{u}}}_{f i}
\end{array}\right\}, \quad V_{e}=\frac{1}{2}\left\{\begin{array}{c}
\boldsymbol{u}_{r} \\
\overline{\boldsymbol{u}}_{f c} \\
\overline{\boldsymbol{u}}_{f i}
\end{array}\right\}^{T}\left[\begin{array}{lll}
\boldsymbol{K}_{11} & \boldsymbol{K}_{12} & \boldsymbol{K}_{13} \\
\boldsymbol{K}_{21} & \boldsymbol{K}_{22} & \boldsymbol{K}_{23} \\
\boldsymbol{K}_{31} & \boldsymbol{K}_{32} & \boldsymbol{K}_{33}
\end{array}\right]\left\{\begin{array}{c}
\boldsymbol{u}_{r} \\
\overline{\boldsymbol{u}}_{f c} \\
\overline{\boldsymbol{u}}_{f i}
\end{array}\right\},
$$

where $\overline{\boldsymbol{u}}_{f c}=\left\{\overline{\boldsymbol{u}}_{f}^{(1) T} \overline{\boldsymbol{u}}_{f}^{(2)}{ }^{T} \overline{\boldsymbol{u}}_{f}^{(3)}{ }^{T} \overline{\boldsymbol{u}}_{f}^{(4)}{ }^{T}\right\}^{T}$ represents the DOFs corresponding to the constrained nodes and $\overline{\boldsymbol{u}}_{f i}$ represents the unconstrained DOFs.

From (3), $\overline{\boldsymbol{u}}_{f c}$ can be expressed in terms of $\boldsymbol{u}_{r}$. The independent DOFs become $\tilde{\boldsymbol{q}}=\left\{\begin{array}{lll}\boldsymbol{u}_{r}^{T} & \overline{\boldsymbol{u}}_{f i}^{T}\end{array}\right\}^{T}$. Substituting into (4), the kinetic energy and elastic potential energy become

$$
\begin{aligned}
T & =\frac{1}{2} \dot{\tilde{\boldsymbol{q}}}^{T}\left[\begin{array}{ll}
\boldsymbol{M}_{11} & \boldsymbol{M}_{13} \\
\boldsymbol{M}_{31} & \boldsymbol{M}_{33}
\end{array}\right] \dot{\tilde{\boldsymbol{q}}}+\bar{T}(\dot{\tilde{\boldsymbol{q}}}, \tilde{\boldsymbol{q}})=\frac{1}{2} \dot{\tilde{\boldsymbol{q}}}^{T} \boldsymbol{M}_{s} \dot{\tilde{\boldsymbol{q}}}+\bar{T}(\dot{\tilde{\boldsymbol{q}}}, \tilde{\boldsymbol{q}}), \\
V_{e} & =\frac{1}{2} \tilde{\boldsymbol{q}}^{T}\left[\begin{array}{ll}
\boldsymbol{K}_{11} & \boldsymbol{K}_{13} \\
\boldsymbol{K}_{31} & \boldsymbol{K}_{33}
\end{array}\right] \tilde{\boldsymbol{q}}+\bar{V}_{e}(\tilde{\boldsymbol{q}})=\frac{1}{2} \tilde{\boldsymbol{q}}^{T} \boldsymbol{K}_{s} \tilde{\boldsymbol{q}}+\bar{V}_{e}(\tilde{\boldsymbol{q}}),
\end{aligned}
$$

where

$$
\begin{aligned}
\bar{T}(\dot{\tilde{\boldsymbol{q}}}, \tilde{\boldsymbol{q}}) & =\frac{1}{2}\left(\dot{\boldsymbol{u}}_{r}^{T} \boldsymbol{M}_{12} \dot{\overline{\boldsymbol{u}}}_{f c}+\dot{\overline{\boldsymbol{u}}}_{f c}^{T} \boldsymbol{M}_{21} \dot{\boldsymbol{u}}_{r}+\dot{\overline{\boldsymbol{u}}}_{f i}^{T} \boldsymbol{M}_{32} \dot{\overline{\boldsymbol{u}}}_{f c}+\dot{\overline{\boldsymbol{u}}}_{f c}^{T} \boldsymbol{M}_{23} \dot{\boldsymbol{u}}_{r}+\dot{\overline{\boldsymbol{u}}}_{f c}^{T} \boldsymbol{M}_{22} \dot{\overline{\boldsymbol{u}}}_{f c}\right), \\
\bar{V} & (\tilde{\boldsymbol{q}}) \\
= & \frac{1}{2}\left(\boldsymbol{u}_{r}^{T} \boldsymbol{K}_{12} \overline{\boldsymbol{u}}_{f c}+\overline{\boldsymbol{u}}_{f c}^{T} \boldsymbol{K}_{21} \boldsymbol{u}_{r}+\overline{\boldsymbol{u}}_{f i}^{T} \boldsymbol{K}_{32} \overline{\boldsymbol{u}}_{f c}+\overline{\boldsymbol{u}}_{f c}^{T} \boldsymbol{K}_{23} \boldsymbol{u}_{r}+\overline{\boldsymbol{u}}_{f c}^{T} \boldsymbol{K}_{22} \overline{\boldsymbol{u}}_{f c}\right) .
\end{aligned}
$$

Gravity potential can also be expressed as a composite function of $\tilde{\boldsymbol{q}}$ :

$$
V_{g}=V_{g}\left[\boldsymbol{u}_{r}, \overline{\boldsymbol{u}}_{f c}\left(\boldsymbol{u}_{r}\right), \overline{\boldsymbol{u}}_{f i}\right]=V_{g}(\tilde{\boldsymbol{q}}) .
$$

Assuming no nonconservative load, the equations of motion of the system can be obtained using the Lagrange equations [Meirovitch 2001]:

$$
\frac{d}{d t}\left(\frac{\partial T}{\partial \dot{\tilde{\boldsymbol{q}}}}\right)^{T}-\left(\frac{\partial T}{\partial \tilde{\boldsymbol{q}}}\right)^{T}+\left(\frac{\partial V_{e}}{\partial \tilde{\boldsymbol{q}}}\right)^{T}+\left(\frac{\partial V_{g}}{\partial \tilde{\boldsymbol{q}}}\right)^{T}=\mathbf{0} .
$$


Substituting (2)-(4) into (6), notice that $\boldsymbol{M}_{s}$ depends on $\tilde{\boldsymbol{q}}$ while $\boldsymbol{K}_{s}$ is a constant. The equations of motion become

$$
\boldsymbol{M}_{s} \ddot{\tilde{\boldsymbol{q}}}+\dot{\boldsymbol{M}}_{s} \dot{\tilde{\boldsymbol{q}}}+\frac{d}{d t}\left(\frac{\partial \bar{T}}{\partial \dot{\tilde{\boldsymbol{q}}}}\right)-\frac{\partial}{\partial \tilde{\boldsymbol{q}}}\left(\frac{1}{2} \dot{\tilde{\boldsymbol{q}}} \boldsymbol{M}_{s} \dot{\tilde{\boldsymbol{q}}}\right)^{T}-\frac{\partial \bar{T}}{\partial \tilde{\boldsymbol{q}}}+\boldsymbol{K}_{s} \tilde{\boldsymbol{q}}+\boldsymbol{R}(\tilde{\boldsymbol{q}})+\boldsymbol{H}(\tilde{\boldsymbol{q}})=\mathbf{0}
$$

where $\boldsymbol{R}$ represents the nonlinear term from the derivative of the elastic potential energy and $\boldsymbol{H}$ represents the force and moment induced by gravity:

$$
\begin{aligned}
& \boldsymbol{R}(\tilde{\boldsymbol{q}})=\frac{\partial \bar{V}_{e}}{\partial \tilde{\boldsymbol{q}}}=\left[\begin{array}{c}
\boldsymbol{K}_{12} \overline{\boldsymbol{u}}_{f c}+\left(\frac{\partial \overline{\boldsymbol{u}}_{f c}}{\partial \boldsymbol{u}_{r}}\right)^{T} \boldsymbol{K}_{21} \boldsymbol{u}_{r}+\left(\frac{\partial \overline{\boldsymbol{u}}_{f c}}{\partial \boldsymbol{u}_{r}}\right)^{T} \boldsymbol{K}_{23} \overline{\boldsymbol{u}}_{f i}+\left(\frac{\partial \overline{\boldsymbol{u}}_{f c}}{\partial \boldsymbol{u}_{r}}\right)^{T} \boldsymbol{K}_{22} \overline{\boldsymbol{u}}_{f c} \\
\boldsymbol{K}_{32} \overline{\boldsymbol{u}}_{f c}
\end{array}\right]=\left[\begin{array}{c}
\boldsymbol{R}_{r} \\
\boldsymbol{R}_{f i}
\end{array}\right], \\
& \boldsymbol{H}(\tilde{\boldsymbol{q}})=\frac{\partial V_{g}}{\partial \tilde{\boldsymbol{q}}}=\left\{\left(\frac{\partial V_{g}}{\partial \boldsymbol{u}_{r}}+\frac{\partial V_{g}}{\partial \overline{\boldsymbol{u}}_{f c}} \frac{\partial \overline{\boldsymbol{u}}_{f c}}{\partial \boldsymbol{u}_{r}}\right)^{T},\left(\frac{\partial V_{g}}{\partial \overline{\boldsymbol{u}}_{f i}}\right)^{T}\right\}^{T},
\end{aligned}
$$

where

$$
\frac{\partial V_{g}}{\partial \boldsymbol{u}_{r}}=\int_{V} \rho g\left[\begin{array}{lll}
0 & 1 & 0
\end{array}\right]\left[\begin{array}{ll}
\boldsymbol{I} & \boldsymbol{B}
\end{array}\right] d V, \quad \frac{\partial V_{g}}{\partial \overline{\boldsymbol{u}}_{f c}}=\int_{V} \rho g\left[\begin{array}{lll}
0 & 1 & 0
\end{array}\right][\tilde{\tilde{\boldsymbol{A}}}] d V,
$$

and $\tilde{\tilde{A}}$ is a fraction of $\tilde{\boldsymbol{A}}$ which corresponds to the constraint DOFs $\boldsymbol{u}_{f c}$. From (3), it can be obtained that

$$
\frac{\partial \overline{\boldsymbol{u}}_{f c}}{\partial \boldsymbol{u}_{r}}=-\boldsymbol{A}^{-1}\left[\begin{array}{ll}
\boldsymbol{I} & \left.\boldsymbol{B}\left(\boldsymbol{u}_{c}^{*}\right)\right]
\end{array}\right.
$$

where $\boldsymbol{u}_{c}^{*}=\boldsymbol{x}+\overline{\boldsymbol{u}}_{f c}(\tilde{\boldsymbol{q}})$.

The damping effect caused by the velocity terms is not within the scope of this paper. Ignoring the velocity terms $\dot{\boldsymbol{M}}_{s} \dot{\tilde{\boldsymbol{q}}},(\partial / \partial \tilde{\boldsymbol{q}})\left(\frac{1}{2} \dot{\tilde{\boldsymbol{q}}} \boldsymbol{M}_{s} \dot{\tilde{\boldsymbol{q}}}\right), \partial \bar{T} / \partial \tilde{\boldsymbol{q}}$, and those in $(d / d t)(\partial \bar{T} / \partial \dot{\tilde{\boldsymbol{q}}})$, the governing equations become

$$
\boldsymbol{M}_{s} \ddot{\tilde{\boldsymbol{q}}}+\boldsymbol{K}_{s} \tilde{\boldsymbol{q}}+\boldsymbol{S}(\ddot{\tilde{\boldsymbol{q}}}, \tilde{\boldsymbol{q}})+\boldsymbol{R}(\tilde{\boldsymbol{q}})+\boldsymbol{H}(\tilde{\boldsymbol{q}})=\mathbf{0}
$$

where

$$
\boldsymbol{S}(\ddot{\tilde{\boldsymbol{q}}}, \tilde{\boldsymbol{q}})=\left[\begin{array}{c}
\boldsymbol{M}_{12} \ddot{\overline{\boldsymbol{u}}}_{f c}+\left(\frac{\partial \overline{\boldsymbol{u}}_{f c}}{\partial \boldsymbol{u}_{r}}\right)^{T} \boldsymbol{M}_{21} \ddot{\boldsymbol{u}}_{r}+\left(\frac{\partial \boldsymbol{u}_{f c}}{\partial \boldsymbol{u}_{r}}\right)^{T} \boldsymbol{M}_{23} \ddot{\overline{\boldsymbol{u}}}_{f i}+\left(\frac{\partial \overline{\boldsymbol{u}}_{f c}}{\partial \boldsymbol{u}_{r}}\right)^{T} \boldsymbol{M}_{22} \ddot{\overline{\boldsymbol{u}}}_{f c} \\
\boldsymbol{M}_{32} \ddot{\overline{\boldsymbol{u}}}_{f c}
\end{array}\right]=\left[\begin{array}{c}
\boldsymbol{S}_{r} \\
\boldsymbol{S}_{f i}
\end{array}\right] .
$$

If the dynamic terms in (7) are dropped, a static equilibrium solution of the system $\tilde{\boldsymbol{q}}^{0}$ can be obtained through iteration. A Fortran90 code is implemented to solve for the equilibrium solution. For a convergence criterion of 0.001 for the L2 norm of the displacement vector, it takes two steps to reach the converged equilibrium solution. Figure 2 shows the scaled global deformation of the bundle at three different locations for the equilibrium solution.

Denoting $\delta$ as the deviation from the equilibrium position, the generalized coordinates can be expressed as $\tilde{\boldsymbol{q}}=\tilde{\boldsymbol{q}}^{0}+\boldsymbol{\delta}$. Substituting this equation into (7), dropping the constant terms and linearizing the nonlinear terms with a Taylor expansion at $\tilde{\boldsymbol{q}}^{0}$, the governing equation for oscillation about the equilibrium can be obtained as

$$
\boldsymbol{M}_{s} \ddot{\boldsymbol{\delta}}+\boldsymbol{S}\left(\ddot{\boldsymbol{\delta}}, \tilde{\boldsymbol{q}}^{0}\right)+\left(\boldsymbol{K}_{s}+\frac{\partial \boldsymbol{R}}{\partial \tilde{\boldsymbol{q}}}\left(\tilde{\boldsymbol{q}}^{0}\right)+\frac{\partial \boldsymbol{H}}{\partial \tilde{\boldsymbol{q}}}\left(\tilde{\boldsymbol{q}}^{0}\right)\right) \boldsymbol{\delta}=\mathbf{0} .
$$


The term $\boldsymbol{S}\left(\ddot{\boldsymbol{\delta}}, \tilde{\boldsymbol{q}}^{0}\right)$ can be further linearized using the Taylor expansion at $\ddot{\boldsymbol{\delta}}=\mathbf{0}$, and (8) becomes

$$
\left(\boldsymbol{M}_{s}+\frac{\partial \boldsymbol{S}}{\partial \ddot{\boldsymbol{\delta}}}\left(\mathbf{0}, \tilde{\boldsymbol{q}}^{0}\right)\right) \ddot{\boldsymbol{\delta}}+\left(\boldsymbol{K}_{s}+\frac{\partial \boldsymbol{R}}{\partial \tilde{\boldsymbol{q}}}\left(\tilde{\boldsymbol{q}}^{0}\right)+\frac{\partial \boldsymbol{H}}{\partial \tilde{\boldsymbol{q}}}\left(\tilde{\boldsymbol{q}}^{0}\right)\right) \boldsymbol{\delta}=\mathbf{0},
$$

where

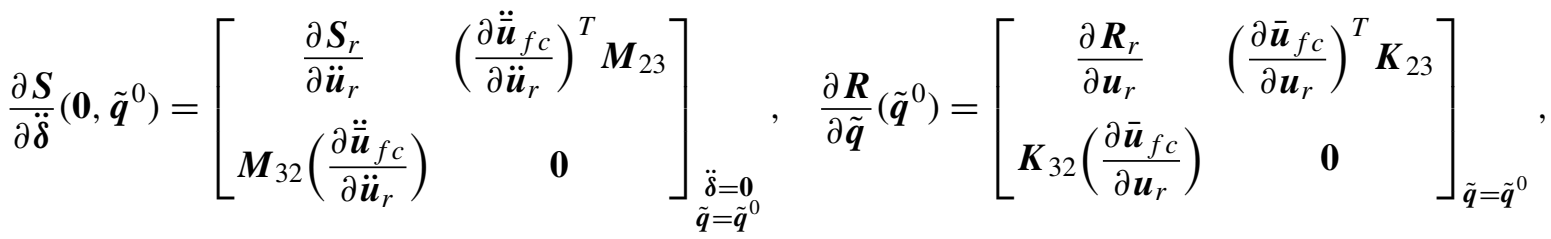

$$
\begin{aligned}
& \frac{\partial \ddot{\boldsymbol{u}}_{f c}}{\partial \ddot{\boldsymbol{u}}_{r}} \doteq\left[\begin{array}{c}
-\boldsymbol{A}^{-1}-\boldsymbol{A}^{-1} \boldsymbol{B}\left(\varphi, \boldsymbol{u}^{(1)}\right) \\
\vdots \\
-\boldsymbol{A}^{-1}-\boldsymbol{A}^{-1} \boldsymbol{B}\left(\varphi, \boldsymbol{u}^{(4)}\right)
\end{array}\right], \quad \frac{\partial \boldsymbol{u}_{f c}}{\partial \boldsymbol{u}_{r}} \doteq\left[\begin{array}{c}
-\boldsymbol{A}^{-1}-\boldsymbol{A}^{-1} \boldsymbol{B}\left(\varphi, \boldsymbol{u}^{*(1)}\right) \\
\vdots \\
-\boldsymbol{A}^{-1}-\boldsymbol{A}^{-1} \boldsymbol{B}\left(\varphi, \boldsymbol{u}^{*(4)}\right)
\end{array}\right], \\
& \frac{\partial \boldsymbol{H}}{\partial \tilde{\boldsymbol{q}}}=\sum_{i=1}^{N e} \int_{V_{i}}\left[\begin{array}{ccc}
\mathbf{0} & \mathbf{0} & \mathbf{0} \\
\mathbf{0} & \frac{\partial \boldsymbol{B}_{2,:}}{\partial \boldsymbol{\phi}} & \frac{\partial \boldsymbol{B}_{2,:}}{\partial \boldsymbol{u}_{f}} \\
\mathbf{0} & \frac{\partial \tilde{\boldsymbol{A}}_{2,:}}{\partial \boldsymbol{\phi}} & \mathbf{0}
\end{array}\right]_{\tilde{\boldsymbol{q}}=\tilde{\boldsymbol{q}}^{0}} d V
\end{aligned}
$$

and where the subscript $(2,:)$ represents the second row of the matrix. $\frac{\partial \boldsymbol{S}_{r}}{\partial \ddot{\boldsymbol{u}}_{r}}$ and $\frac{\partial \boldsymbol{R}_{r}}{\partial \boldsymbol{u}_{r}}$ are $6 \times 6$ matrices.

\section{Numerical solutions and discussion}

A numerical solution to the governing equations of motion, (9), is sought. The geometry and material properties of the bundle are listed in Table 1. The numerical solution shows that the fundamental natural frequency is $6.1 \mathrm{~Hz}$. After normalizing the eigenvector, it is found that the $Z$-direction rigid body rotation is the primary dominant component and the $X$-direction rigid body translation is the secondary. The
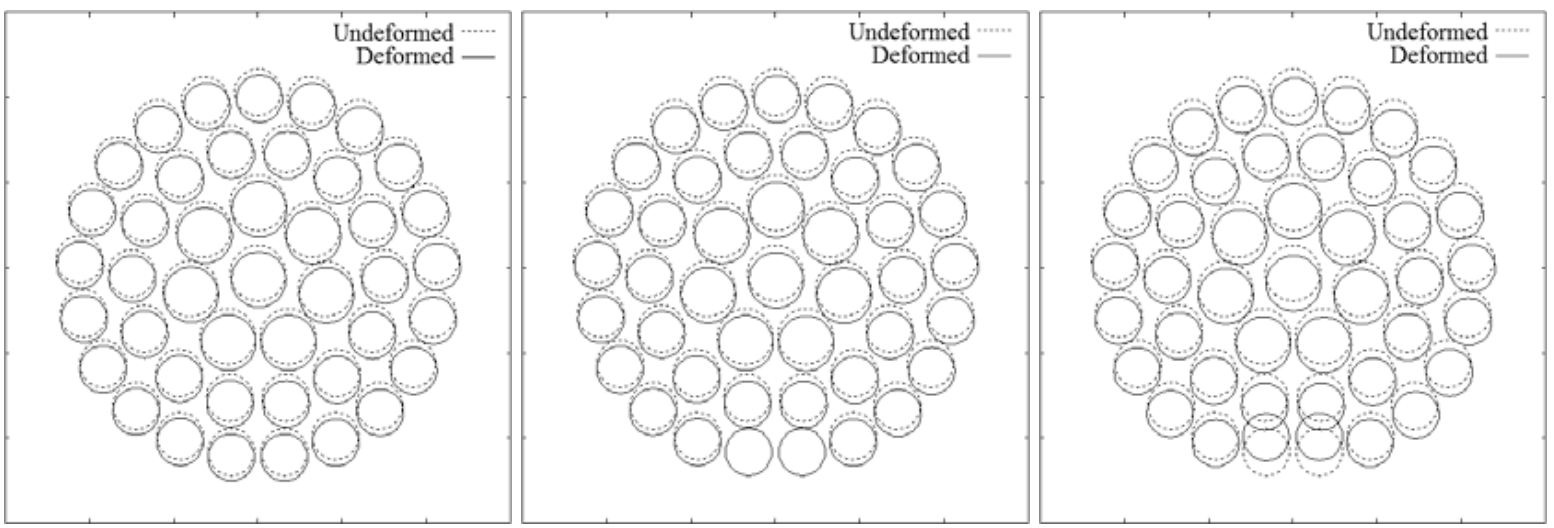

Figure 2. Scaled deformation in equilibrium at different locations: bundle ends (left), bearing pads (middle), and midspan (right). 


\begin{tabular}{|lll|}
\hline Parameter & Symbol & Value \\
Bundle length $(\mathrm{mm})$ & $L$ & 500.0 \\
Outer ring mean radius $(\mathrm{mm})$ & $R_{1}$ & 42.5 \\
Intermediate ring mean radius $(\mathrm{mm})$ & $R_{2}$ & 30.0 \\
Inner ring mean radius $(\mathrm{mm})$ & $R_{3}$ & 16.5 \\
Ring and rib width $(\mathrm{mm})$ & $b$ & 3.4 \\
Endplate thickness $(\mathrm{mm})$ & $t$ & 3.0 \\
Young's modulus $(\mathrm{GPa})$ & $E$ & 200.0 \\
Poisson's ratio & $v$ & 0.3 \\
Shear modulus $(\mathrm{GPa})$ & $G$ & 76.9 \\
\hline
\end{tabular}

Table 1. Geometric dimensions and material properties.
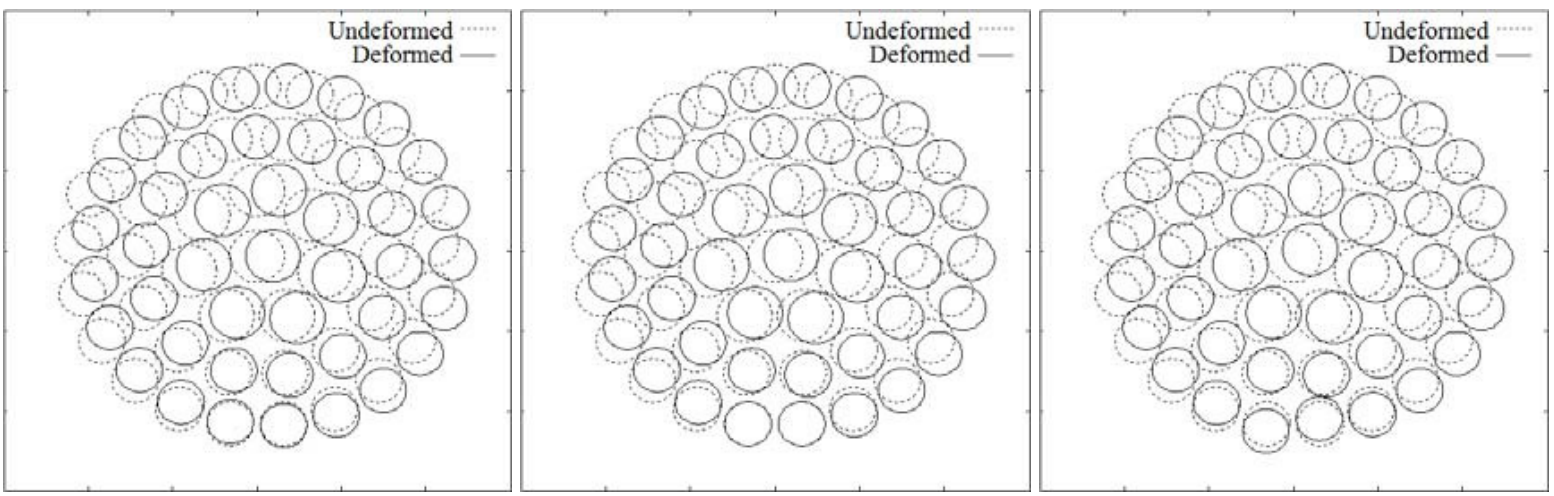

Figure 3. Scaled deviation of the rocking mode at different locations: bundle ends (left), bearing pads (middle), and midspan (right).

elastic DOFs are negligibly small except those on the two supporting rods at the bottom. To visualize the solution, the mode shape of the small deviation is shown in Figure 3. The total displacement, which is the static deformation superposed with the scaled deviation eigenvector, is shown in Figure 4. The mode exhibits a rocking motion in terms of the total displacement.

It is necessary to validate the accuracy of the meshing scheme and the numerical methods used in this paper against independent finite element code. Most general finite element codes do not incorporate the floating frame formulation; therefore comparison can be made on a conventional finite element model of the bundle structure. The above rigid-elasto model is degraded to a conventional finite element model by removing the floating frame formulation. The modal solution from this model is compared to that of an independent finite element model developed in ANSYS ${ }^{\circledR}$ ED 8.0 using straight beam elements. The comparison of the natural frequencies is shown in Table 2.

From the above solutions, it can be seen that the rigid body motion and gravity have a significant influence on the rocking frequency. The structural solution, which does not include the rigid body DOFs and the gravity terms, shows a frequency of $7.7 \mathrm{~Hz}$, while the rigid-elasto solution shows a frequency of 

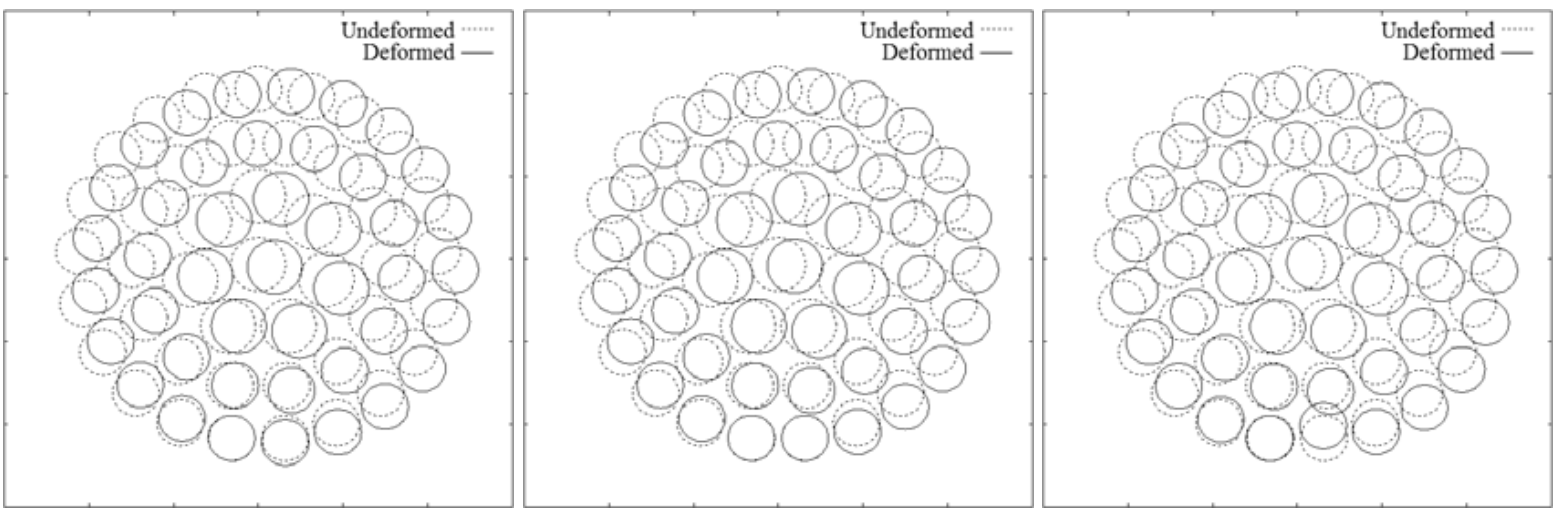

Figure 4. Scaled total displacement of the rocking mode at different locations: bundle ends (left), bearing pads (middle), and midspan (right).

\begin{tabular}{|ccc|}
\hline Mode & \multicolumn{2}{c|}{ Natural frequencies $(\mathrm{Hz})$} \\
& This paper & ANSYS ED 8.0 \\
1 & 7.7 & 7.5 \\
2 & 50.8 & 50.0 \\
3 & 93.0 & 87.7 \\
4 & 93.8 & 93.2 \\
5 & 94.3 & 93.6 \\
6 & 94.8 & 94.0 \\
\hline
\end{tabular}

Table 2. Validation on the current finite element model (without floating frame formulation) against ANSYS ED 8.0.

\begin{tabular}{|cccc|}
\hline Mode & \multicolumn{2}{c}{ Frequencies $(\mathrm{Hz})$} & Difference $(\%)$ \\
& With & Without & \\
1 & 6.1 & 7.7 & 20.8 \\
2 & 50.4 & 50.8 & 0.8 \\
3 & 91.9 & 93.0 & 1.2 \\
4 & 93.8 & 93.8 & 0.0 \\
5 & 94.2 & 94.3 & 0.1 \\
6 & 94.4 & 94.8 & 0.4 \\
\hline
\end{tabular}

Table 3. Influence of gravity. Frequencies are shown for with and without floating frame formulation and gravity.

6.1 Hz. The difference is $20.8 \%$. It can be seen in Table 3 that the influence of gravity is only significant on the fundamental mode. This is because the fundamental mode is related to rigid body rotations and gravity does positive work when the bundle rocks from the equilibrium position to either side. The 

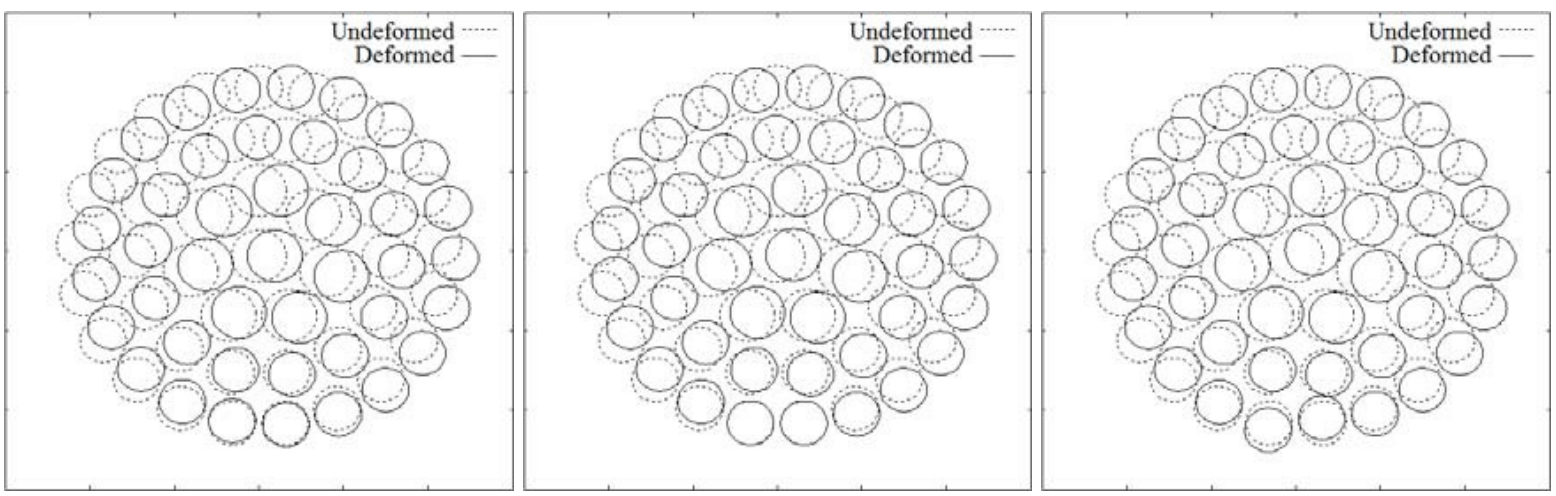

Figure 5. Scaled deformation of the rocking mode at different locations without floating frame formulation and nonlinear terms: bundle ends (left), bearing pads (middle), and midspan (right).

second mode is a pure vertical motion and the gravity work cancels itself in the motion. Higher modes are characterized by the bending deformation of the rods, and thus receive little influence from gravity. The fundamental mode of the structural solution is also shown in Figure 5 for comparison.

Although the influence of gravity is important, it needs to be clarified that the impact is delivered "indirectly". The major influence of gravity is enforced through the equilibrium solution hidden in the nonlinear terms $\partial \boldsymbol{S} / \partial \ddot{\boldsymbol{\delta}}$ and $\partial \boldsymbol{R} / \partial \tilde{\boldsymbol{q}}$, especially the latter. The $\partial \boldsymbol{R}_{r} / \partial \boldsymbol{u}_{r}$ term in $\partial \boldsymbol{R} / \partial \tilde{\boldsymbol{q}}$ can be further expressed as

$$
\begin{array}{r}
\frac{\partial \boldsymbol{R}_{r}}{\partial \boldsymbol{u}_{r}}=\left[\boldsymbol{K}_{12} \frac{\partial \boldsymbol{u}_{f c}}{\partial \boldsymbol{u}_{r}}+\left(\frac{\partial \boldsymbol{u}_{f c}}{\partial \boldsymbol{u}_{r}}\right)^{T} \boldsymbol{K}_{21}+2\left(\frac{\partial \boldsymbol{u}_{f c}}{\partial \boldsymbol{u}_{r}}\right)^{T} \boldsymbol{K}_{22} \frac{\partial \boldsymbol{u}_{f c}}{\partial \boldsymbol{u}_{r}}\right]_{\tilde{\boldsymbol{q}}^{0}} \\
+\left[\boldsymbol{u}_{r}^{T} \frac{\partial \boldsymbol{h}_{i}^{A}}{\partial \boldsymbol{u}_{r}}+\frac{\partial}{\partial \boldsymbol{u}_{r}}\left(\boldsymbol{h}_{i}^{B} \boldsymbol{u}_{f i}\right)+\boldsymbol{u}_{r}^{T} \frac{\partial \boldsymbol{h}_{i}^{C}}{\partial \boldsymbol{u}_{r}}\right]_{\tilde{\boldsymbol{q}}^{0}},
\end{array}
$$

where $\boldsymbol{h}_{i}^{A}, \boldsymbol{h}_{i}^{B}$, and $\boldsymbol{h}_{i}^{C}$ are the $i$-th rows of $\left(\partial \boldsymbol{u}_{f c} / \partial \boldsymbol{u}_{r}\right)^{T} \boldsymbol{K}_{21},\left(\partial \boldsymbol{u}_{f c} / \partial \boldsymbol{u}_{r}\right)^{T} \boldsymbol{K}_{23}$, and $\left(\partial \boldsymbol{u}_{f c} / \partial \boldsymbol{u}_{r}\right)^{T} \boldsymbol{K}_{22}$, respectively. The terms in the second set of brackets in (10) contain the equilibrium solution which is a consequence of gravity. If this term is dropped, the rocking frequency will increase to a value which is almost identical to the structural solution. It should be noticed that the coupling term between the constraint DOF and the rigid body DOF terms $\boldsymbol{K}_{12}$ and $\boldsymbol{K}_{21}$ is actually zero; therefore, the corresponding terms in the second set of brackets are dominant. Even a small quantity in the second set of brackets may have a strong influence on the result. Dissimilarly to this, the contributions from the nonlinear terms in $\partial \boldsymbol{S} / \partial \ddot{\boldsymbol{\delta}}$ are very small compared to those from $\boldsymbol{M}_{12}$ and $\boldsymbol{M}_{21}$, and hence have little influence on the result.

Influences from the nonlinearity in the Euler rotation matrix $\boldsymbol{A}$, the derivative matrix $\boldsymbol{B}$, and the constraint equations are relatively small compared to the indirect influence of gravity. However, if the constraint equations are linearized so that $\partial \boldsymbol{u}_{f c} / \partial \boldsymbol{u}_{r}$ becomes a constant, then the derivatives of $\boldsymbol{h}_{i}^{A}, \boldsymbol{h}_{i}^{B}$, and $\boldsymbol{h}_{i}^{C}$ in (10) will become zero and hence the influence from gravity will be lost. 
There is also a "direct" influence of gravity, the $\partial \boldsymbol{H} / \partial \tilde{\boldsymbol{q}}$ term. This represents the rate of change in the gravity-induced moment with respect to the displacement. Although this term is derived from the gravity potential, its impact is negligible in this application, because the rods in the bundle are almost evenly distributed. Numerical solution shows that the values of the elements in the $\partial \boldsymbol{H} / \partial \tilde{\boldsymbol{q}}$ term are a few orders lower in magnitude then those in the $\boldsymbol{K}_{s}$ and $\partial \boldsymbol{R} / \partial \tilde{\boldsymbol{q}}$ terms.

\section{Experiment}

An experiment was carried out to determine the rocking frequency of the bundle. Lateral oscillation in the horizontal direction of the bundle may be related to the rocking motion of the bundle. Its frequency can be obtained by monitoring the oscillatory motion of a point on the outer ring of the bundle near the end. Instead of using accelerometers, a noncontact displacement sensor is used for vibration measurement. This type of sensor is more sensitive to low frequency, relatively large displacement vibrations.

To measure the bundle vibration, a 43-rod bundle is placed inside a 4-inch PVC tube supported by strong steel columns, as shown in Figure 6. A noncontact differential variable reluctance transducer (MicroStrain NC-DVRT-1.5) is used to recode the lateral displacement of the bundle. The sensor detects the distance between a target object and the face of the transducer head. The reluctance of the coils within the sensor is changed when the face of the transducer is in close proximity to a ferrous or highly conductive material. The transducer outputs a voltage signal that is a nonlinear function of the distance. The typical repeatability of this transducer is $\pm 2 \mu \mathrm{m}$ and the frequency range is 0 to $800 \mathrm{~Hz}$. The transducer is mounted at one side of the tube near the end of the bundle. The transducer head penetrates the tube wall and approaches the rod around the 4 o'clock position. The initial gap from the head of the transducer is around $200 \mu \mathrm{m}$ for the best gain factor in the output. The time-domain voltage signal is sampled with a sampling rate of $5 \mathrm{kHz}$ and recorded using a data acquisition system. The voltage signal is then converted to gap distance and analyzed using code written in Matlab to obtain the spectral information.
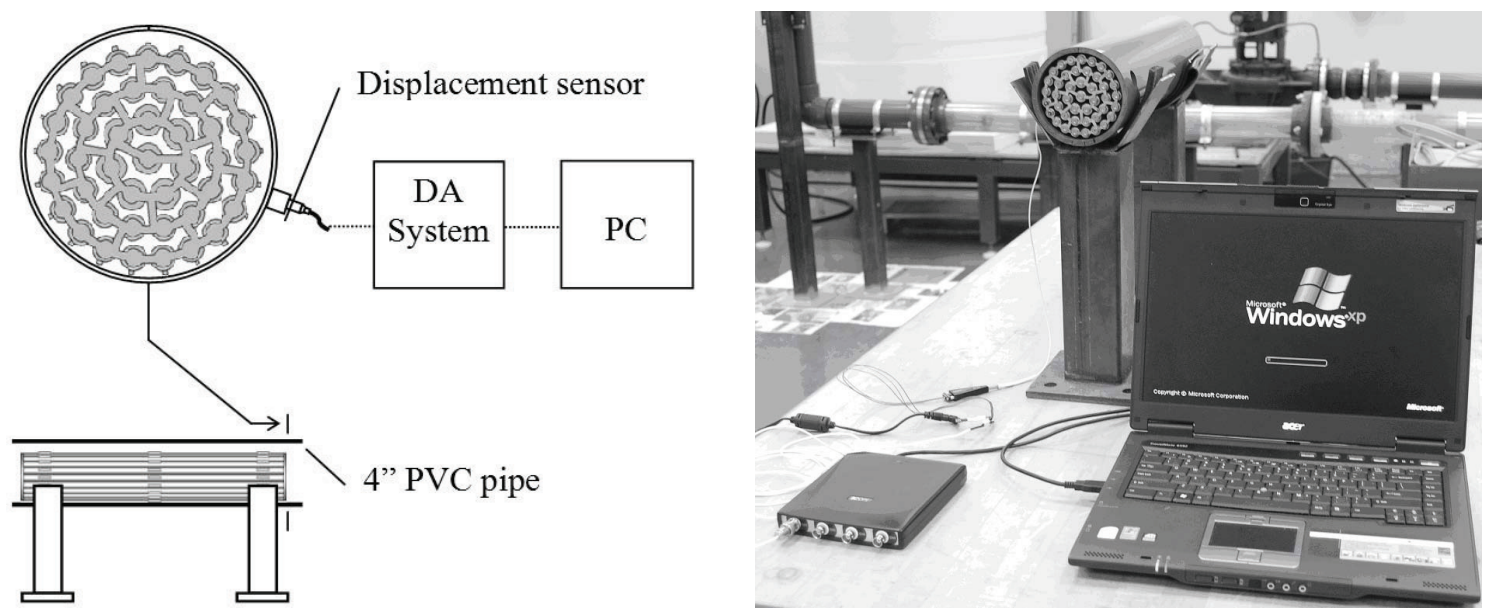

Figure 6. Experimental setup. 

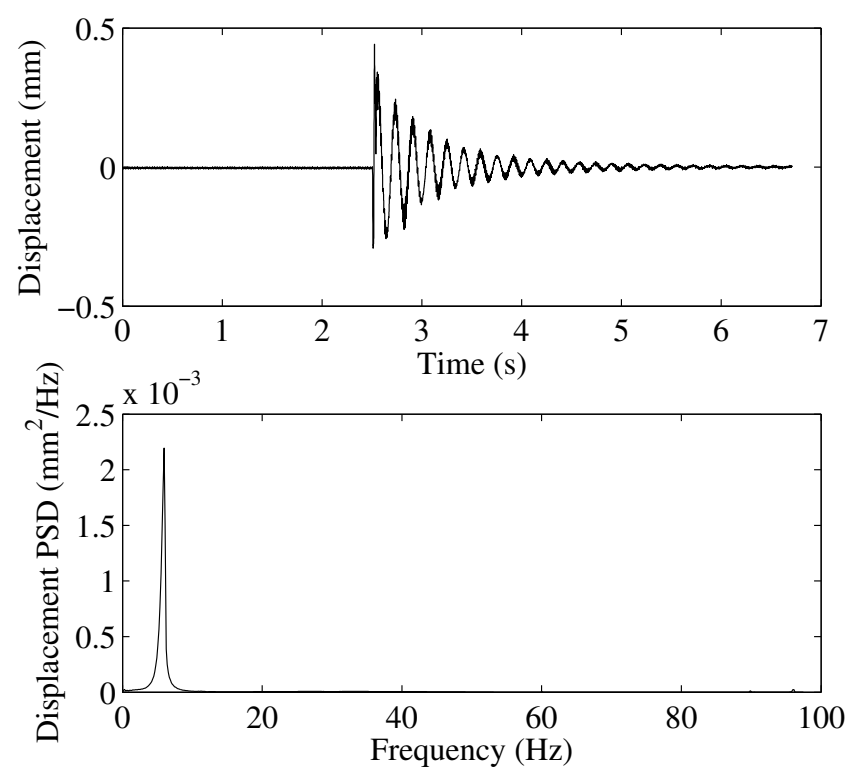

Figure 7. The response of the bundle to an impulse.

The response of the bundle to an impulse is recorded and analyzed. The time-domain displacement of the monitored point and its power spectral density are shown in Figure 7. The response of the rocking mode is found to be clear and free of noise from higher modes, such as the $90-100 \mathrm{~Hz}$ mode group corresponding to the first bending mode of the rods. The rocking mode frequency is $6.0 \mathrm{~Hz}$. The damping ratio calculated from the difference between the peaks in the time-domain signal is about 0.05 . For such a low damping ratio, the effect of damping on natural frequencies is negligible.

The tube is built from PVC, a material softer than steel. When the steel bearing pads contact the tube inner surface, the soft material will deform. This deformation leads to a contact stiffness and may have an influence on the fundamental frequency. It is necessary to examine the contact stiffness and compare it to the overall equivalent stiffness that relates to the bundle deformation with the current boundary conditions. The Boussinesq point contact solution [Johnson 1985] is used to estimate the order of magnitude of the normal contact stiffness $k_{C}$. Based on the solution, the normal elastic deformation $w$ at the contact location when a point object indents on a semiinfinite elastic space in the normal direction can be expressed as

$$
w=\frac{2 P(1-v)}{4 \pi G r},
$$

where $P$ is the normal contact force, $r$ is the contact point radius, $G$ is the shear modulus of the elastic space ( $G=1 \mathrm{GPa}$ for PVC), and $v$ is the Poisson's ratio of the elastic space ( $v=0.41$ for PVC). The order of magnitude of the contact stiffness on one bearing pad can then be estimated as $k_{C, 1} \sim P / w=$ $\pi G r /(1-v) \approx 10^{7}$. The overall contact stiffness provided by the contact of the four bearing pads is $k_{C}=4 k_{C, 1} \sim 4 \times 10^{7}$. Because the most potential energy in the rocking mode comes from the deformation of the two rods that contact the tube through bearing pads, it is reasonable to compare $k_{C}$ with the equivalent stiffness of these rods. The equivalent stiffness can be conveniently estimated by dividing the weight of the bundle and the vertical displacement of its mass center from a static analysis. 
The order of magnitude of the equivalent stiffness is obtained as $k_{E} \sim 1 \times 10^{6}$, which is far smaller than $k_{C}$. This indicates that the contact stiffness can be considered infinite and the tube can be regarded as rigid with regards to the lower bundle vibration modes.

\section{Conclusions}

The vibration of a 43-rod simulation CANDU fuel bundle horizontally placed in a supporting tube is studied through numerical models and experiments. The floating frame formulation and nonlinear constraint conditions are employed in a finite element model to predict the natural frequencies of the small-amplitude oscillations about the equilibrium position. The fundamental mode is found to be a low frequency rocking mode, which is a combination of rigid body motion and elastic deformation, but is dominated by the rigid body rotation about a bundle axis. Gravity is found to influence the rocking motion, and to reduce the frequency. The simulation results are in good agreement with experimental results.

\section{References}

[Cho et al. 2000] M.-S. Cho, K.-S. Sim, H. C. Suk, and S.-K. Chang, "Static strength analysis of CANDU-6 reactor fuel bundle", Nucl. Eng. Des. 200:3 (2000), 407-419.

[Horhoianu and Ionescu 2006] G. Horhoianu and D. V. Ionescu, "A finite element model for static strength analysis of CANDU fuel bundle", Kerntechnik 71:4 (2006), 203-207.

[Johnson 1985] K. L. Johnson, Contact mechanics, Cambridge University Press, London, 1985.

[Judah 1992] J. Judah, "Overview of fuel inspections at the Darlington nuclear generating station", pp. 1-22 in 3rd International CNS CANDU Fuel Conference (Chalk River, 1992), Canadian Nuclear Society, Toronto, ON, 1992.

[Meirovitch 2001] L. Meirovitch, Fundamentals of vibrations, McGraw-Hill, Boston, 2001.

[Reddy 1984] J. N. Reddy, "A simple higher-order theory for laminated composite plates", J. Appl. Mech. (ASME) 51:4 (1984), 745-752.

[Schwab and Meijaard 2002] A. L. Schwab and J. P. Meijaard, "Small vibrations superimposed on a prescribed rigid body motion", Multibody Syst. Dyn. 8:1 (2002), 29-49.

[Shabana 2005] A. A. Shabana, Dynamics of multibody systems, 3rd ed., Cambridge University Press, Cambridge, 2005.

[Yu and Wen 2007] S. D. Yu and D. C. Wen, "An efficient plate finite element and its application to three-dimensional deformations of CANDU fuel endplate rings", Nucl. Eng. Des. 237:4 (2007), 342-352.

[Zhang and Yu 2010a] X. Zhang and S. D. Yu, "Theoretical and experimental investigations of oscillatory rolling motion of a rod bundle inside a tube", in Proceedings of 11th Pan-American Congress of Applied Mechanics (PACAM XI) (Foz do Iguaçu, 2010), edited by A. R. Aguiar and T. L. Attard, Brazilian Society of Mechanical Sciences and Engineering, Rio de Janeiro, 2010. Paper \#PAC0512.

[Zhang and Yu 2010b] X. Zhang and S. D. Yu, "A thick plate model for bending and twisting of CANDU fuel endplates", Nucl. Eng. Des. 240:10 (2010), 2565-2570.

Received 5 Mar 2010. Revised 3 Jun 2010. Accepted 12 Nov 2010.

XUAN ZHANG: x8zhang@ryerson.ca

Department of Mechanical and Industrial Engineering, Ryerson University, 350 Victoria Street, Toronto, ON M5B 2K3, Canada

SHUDONG YU: syu@ryerson.ca

Department of Mechanical and Industrial Engineering, Ryerson University, 350 Victoria Street, Toronto, ON M5B 2K3, Canada 


\title{
JOURNAL OF MECHANICS OF MATERIALS AND STRUCTURES
}

\author{
jomms.org
}

Founded by Charles R. Steele and Marie-Louise Steele

EDITORS

Charles R. SteEle

DAVIDE BIGONI

Stanford University, USA

YASUHIDE SHINDO

University of Illinois at Urbana-Champaign, USA

Tohoku University, Japan

\section{EDITORIAL BOARD}

$\begin{aligned} \text { H. D. BUI } & \text { École Polytechnique, France } \\ \text { J. P. CARTER } & \text { University of Sydney, Australia } \\ \text { R. M. CHRISTENSEN } & \text { Stanford University, USA } \\ \text { G. M. L. GLADWELL } & \text { University of Waterloo, Canada } \\ \text { D. H. HODGES } & \text { Georgia Institute of Technology, USA } \\ \text { J. HUTCHINSON } & \text { Harvard University, USA } \\ \text { C. HWU } & \text { National Cheng Kung University, Taiwan } \\ \text { B. L. KARIHALOO } & \text { University of Wales, UK } \\ \text { Y. Y. KIM } & \text { Seoul National University, Republic of Korea } \\ \text { Z. MROZ } & \text { Academy of Science, Poland } \\ \text { D. PAMPLONA } & \text { Universidade Católica do Rio de Janeiro, Brazil } \\ \text { M. B. RUBIN } & \text { Technion, Haifa, Israel } \\ \text { A. N. SHUPIKOV } & \text { Ukrainian Academy of Sciences, Ukraine } \\ \text { T. TARNAI } & \text { University Budapest, Hungary } \\ \text { F. Y. M. WAN } & \text { University of California, Irvine, USA } \\ \text { P. WRIGGERS } & \text { Universität Hannover, Germany } \\ \text { W. YANG } & \text { Tsinghua University, China } \\ \text { F. ZIEGLER } & \text { Technische Universität Wien, Austria } \\ & \\ \text { PRODUCTION } & \text { contact@ msp.org } \\ \text { SILVIO LEVY } & \text { Scientific Editor }\end{aligned}$

Cover design: Alex Scorpan

Cover photo: Mando Gomez, www.mandolux.com

See http://jomms.org for submission guidelines.

JoMMS (ISSN 1559-3959) is published in 10 issues a year. The subscription price for 2011 is US \$520/year for the electronic version, and \$690/year (+\$60 shipping outside the US) for print and electronic. Subscriptions, requests for back issues, and changes of address should be sent to Mathematical Sciences Publishers, Department of Mathematics, University of California, Berkeley, CA 94720-3840.

JoMMS peer-review and production is managed by EditFLow ${ }^{\circledR}$ from Mathematical Sciences Publishers.

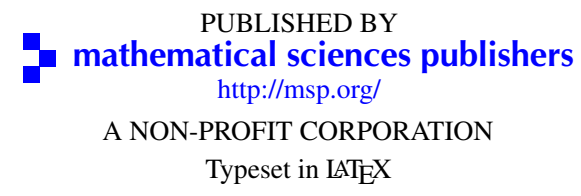

Copyright (C2011 by Mathematical Sciences Publishers 


\section{Journal of Mechanics of Materials and Structures}

\section{Volume 6, No. 7-8}

September-October 2011

\section{Special issue \\ Eleventh Pan-American Congress \\ of Applied Mechanics (PACAM XI)}

Preface

Adair R. Aguiar

949

Influence of specimen geometry on the Portevin-Le Châtelier effect due to dynamic strain aging

for the AA5083-H116 aluminum alloy

Rodrigo Nogueira de Codes and Ahmed Benallal

Dispersion relations for SH waves on a magnetoelectroelastic heterostructure with imperfect

interfaces

J. A. Otero, H. Calas, R. Rodríguez, J. Bravo, A. R. Aguiar and G. Monsivais

Numerical linear stability analysis of a thermocapillary-driven liquid bridge with magnetic stabilization

Yue Huang and Brent C. Houchens

Numerical investigation of director orientation and flow of nematic liquid crystals in a planar 1:4 expansion Pedro a. Cruz, Murilo F. Tomé, IAin W. Stewart and Sean McKee

Critical threshold and underlying dynamical phenomena in pedestrian-induced lateral vibrations of footbridges

Stefano LenCI and LAURA MARCHEgGiani

Free vibration of a simulation CANDU nuclear fuel bundle structure inside a tube

XUAN ZHANG and SHUdONG Yu

Nonlinear dynamics and sensitivity to imperfections in Augusti's model

D. Orlando, P. B. Gonçalves, G. Rega and S. LenCi

Active control of vortex-induced vibrations in offshore catenary risers: A nonlinear normal mode approach

CArlos E. N. MAZzilli and César T. SANCheS

Nonlinear electromechanical fields and localized polarization switching of piezoelectric macrofiber composites

Yasuhide Shindo, Fumio Narita, KoJi SATo and Tomo TAKeda

1089

Three-dimensional BEM analysis to assess delamination cracks between two transversely isotropic materials

Nicolás O. Larrosa, Jhonny E. Ortiz and Adrián P. Cisillino

Porcine dermis in uniaxial cyclic loading: Sample preparation, experimental results and modeling

A. E. Ehret, M. Hollenstein, E. MAzzA and M. Itskov

Analysis of nonstationary random processes using smooth decomposition

Rubens SAMpaio and Sergio Bellizzi

Perturbation stochastic finite element-based homogenization of polycrystalline materials

S. LePage, F. V. Stump, I. H. Kim and P. H. Geubelle

A collocation approach for spatial discretization of stochastic peridynamic modeling of fracture

Georgios I. Evangelatos and POL D. SPANOS 